\title{
ENFOQUES DE APRENDIZAJE EN RESIDENTES DE LA ESPECIALIDAD EN MEDICINA FAMILIAR EN LA DELEGACIÓN GUANAJUATO
}

\author{
Miguel Orlando Cueto-Aguilar ${ }^{*}$, Raúl Hernández-Ordóñez ${ }^{2}$ y Ma. del Pilar Pacheco-Zavala ${ }^{3}$ \\ 'Médico Familiar, Unidad de Medicina Familiar n. ${ }^{0} 56$ del Instituto Mexicano del Seguro Social; ${ }^{2}$ Maestro en Educación, Director Centro de \\ Investigación Educativa y Formación Docente Guanajuato; ${ }^{3}$ Maestra en Ciencias Médicas, Médico familiar, Unidad de Medicina Familiar n. ${ }^{\circ} 53$ del \\ Instituto Mexicano del Seguro Social. Educación Médica. Enfermedades Crónico Degenerativas y Medicina Familiar, León, Guanajuato Gto., México
}

RESUMEN: Introducción: Los enfoques de aprendizaje son procesos que emergen de la percepción que tienen los estudiantes sobre sus tareas académicas, que implican elementos personales e institucionales; se clasifican en superficiales, estratégicos y profundos. Se sabe que a mayor profundidad del enfoque, mejor capacidad para resolver problemas. Objetivo: Determinar los enfoques de aprendizaje en los médicos residentes de la especialidad en Medicina familiar de la Delegación Guanajuato y su asociación con variables sociodemográficas. Material y métodos: Estudio transversal analítico, realizado en 131 residentes de Medicina familiar del ciclo 2017-2018. Se estudió al total de la población, excluyéndose aquellos que estuvieran de vacaciones, licencia o en el servicio social. Se utilizó el Cuestionario R-SPQ2F. Se emplearon medidas de tendencia central para variables cuantitativas, tablas de frecuencia para cualitativas y Chi cuadrada para evaluar asociación entre variables. Resultados: El promedio de edad fue $29.8 \pm 3.1$ años; $58.5 \%$ mujeres y $41.5 \%$ hombres; el $80 \%$ con formación previa en escuelas públicas; un $45.4 \%$ de primer grado, un $37.7 \%$ de segundo y un $16.9 \%$ de tercero. En el $93 \%$ se identificó enfoque profundo, en el $4.7 \%$ superficial y en el $2.3 \%$ mixto. No se encontró asociación entre el enfoque y las variables sociodemográficas grado escolar, sexo y edad. Se evidencia mayor enfoque profundo en aquellas sedes en las que cuentan con todos los servicios y se evita la rotación por sedes alternas (enfoque profundo, $\mathrm{p}=0.005 \mathrm{y}$ enfoque superficial, $\mathrm{p}=0.032$ ). Conclusiones: El enfoque prevalente fue el profundo. En la sede sin rotación externa se encontró únicamente el enfoque profundo.

Palabras clave: Enfoques de aprendizaje. Residencia médica. Medicina familiar.

\section{LEARNING APPROACHES IN RESIDENTS IN FAMILY MEDICINA SPECIALITY AT THE GUANAJUATO DELEGATION}

ABSTRACT: Introduction: Learning approaches are processes that emerge from the perception that students have about their academic tasks and involve personal and institutional elements. They are classified as superficial, strategic and deep. It is known that the deeper the focus, the better the ability to solve problems. Objective: To determine the learning approaches in the residents in Family Medicine in Specialty in Guanajuato Delegation and its association with sociodemographic variables. Methods: Analytical cross-sectional study, carried out in 131 residents of Family Medicine of the 2017-2018 cycle. The total population was studied, excluding those who were on vacation, leave or social service. The Questionnaire R-SPQ- 2 F was used to determine the approaches. We used measures of central tendency for quantitative variables, frequency tables for qualitative and Chi square to evaluate association between variables. Results: The average age was $29.8 \pm 3.1$ years; $58.5 \%$ women, $41.5 \%$ men; $80 \%$ with previous training in public schools; $45.4 \%$ first grade, $37.7 \%$ second grade and $16.9 \%$ third grade. In $93 \%$ a deep focus was identified, $4.7 \%$ with superficial and $2.3 \%$ mixed. No association was found between the approach and the sociodemographic variables, school grade, sex and age. There is a greater deep focus in those offices where they have all the services and rotation by alternate sites is avoided (deep focus, $\mathrm{p}=0.005$, and superficial focus, $\mathrm{p}=0.032$ ). Conclusions: The prevalent approach was the deep one. At headquarters without external rotation only the deep focus was found. (Rev Mex Med Fam. 2019;6:21-7)

Corresponding author: Miguel Orlando

Cueto-Aguilar, orlandocueto@hotmail.com

Key words: Approaches to learning. Medical residency. Family medicine.

\section{Correspondencia:}

*Miguel Orlando Cueto-Aguilar

E-mail: orlandocueto@hotmail.com
Fecha de recepción: 02-08-2018

Fecha de aceptación: 24-11-2018

DOI: 10.24875/RMF.18000101 


\section{INTRODUCCIÓN}

Los estudios sobre enfoques de aprendizaje tuvieron origen en Suecia, donde Marton y Säljö los iniciaron en 1976². En 1987 Biggs los definió como «los procesos que surgen de las percepciones que los estudiantes tienen de las tareas académicas, influidas por sus características personales» ${ }^{2}$. En 1988 Entwistle planteó que el enfoque de aprendizaje es una manera de abordar un contenido de estudio ${ }^{3}$.

Los autores Biggs, Kember y Leung coinciden en señalar que los enfoques de aprendizaje están formados por dos componentes: motivaciones sobre las intenciones que mueven al estudiante a estudiar y estrategias coherentes con dichas intenciones.

Bajo esta perspectiva, las aproximaciones al aprendizaje no son algo estable en el alumno, sino que se relacionan en gran medida con las tareas académicas a las que se enfrente, describiendo la combinación entre la intención y el proceso utilizado por los alumnos para abordar una tarea particular en un momento determinado. Por ello, «un enfoque de aprendizaje describe la naturaleza de la relación entre alumno, contexto y tarea ${ }^{2}$.

Se describen tres dimensiones que caracterizan cómo se aproxima el alumno a sus tareas de aprendizaje: enfoque superficial, profundo y estratégico ${ }^{4}$.

En el enfoque superficial hay implícita una motivación extrínseca e instrumental, donde el alumno pretende satisfacer los requisitos de la tarea con el mínimo esfuerzo, evitando el fracaso. Utiliza como estrategias la memorización y reproducción mecánica, limitándose a lo esencial, centrándose en aspectos concretos y literales, evitando establecer relaciones entre los temas ${ }^{2}$. Es frecuente el uso de fuentes factuales de información, relacionándose con una actitud negativa hacia estas, hay dificultad para dar sentido a ideas nuevas, deficiencia para observar la estructura de los principios inmersos en el material a aprender y escasa actitud reflexiva hacia la tarea. Se ha vinculado a un control firme del maestro en el aprendizaje del alumno y una evaluación que privilegia la memoria. El autoconcepto académico de estos alumnos bastante pobre y suelen estar insatisfechos con su trabajo al comparar sus resultados con los de sus compañeros ${ }^{1}$.

El enfoque profundo se define como una combinación de intenciones y procesos de pensamiento asociados a relacionar ideas y usar la evidencia. Los alumnos tienen alto interés intrínseco y grado de implicación en lo que están aprendiendo; la intención de comprender los lleva al uso de estrategias para descubrir el significado de lo que se trata de aprender y relacionarlo con conocimientos previos relevantes. Este enfoque está relacionado con un alto nivel cualitativo de aprendizaje, conduce a los alumnos a un entendimiento comprensivo, favoreciendo el desarrollo de habilidades de solución de problemas, mayor dominio del contenido, inferencias de alta calidad, autoevaluación-motivación y resultados emocionales positivos ${ }^{2}$.

El enfoque de logro o estratégico se basa en un modo específico de motivación intrínseca-extrínseca como el incremento de la autoestima resultado de un logro académico. El objetivo es manifestar la propia competencia con respecto a los compañeros, intentando obtener las máximas calificaciones. Los alumnos que siguen este enfoque privilegian la autodisciplina, distribución del tiempo, planificación, sistematización y el orden. No suelen presentar problemas de aprendizaje. Sin embargo, su excesivo afán por conseguir elevadas calificaciones puede producirles problemas físicos, psíquicos y sociales ${ }^{2}$.

\section{ANTECEDENTES CIENTÍFICOS}

Los enfoques de aprendizaje han sido investigados en diferentes áreas formativas, sin embargo, su exploración en el área de la salud aún es limitada y más aún en los cursos de especialización médica.

García Perdomo y García de la $\mathrm{Hoz}^{5}$ investigaron el tipo de enfoque en estudiantes de medicina en Cali, Colombia, y 
encontraron que la mayoría de ellos tenía un enfoque profundo, aunque el 25\% presentó un enfoque superficial. En otro estudio realizado en la Escuela de Ciencias de la Salud, de la Universidad de León, España, se encontró que los enfoques de aprendizaje van cambiando a medida que los cursos avanzan, incrementándose el enfoque profundo y disminuyendo el superficial; este efecto se atribuye a una multitud de variables, como: edad, madurez progresiva, contexto educativo, metodologías, actividades y evaluación, entre otras ${ }^{6}$.

Estudios en otras áreas del conocimiento también han reportado un predominio por el enfoque profundo, como el realizado por Hernández Pina, et al., quienes encontraron enfoque profundo en el 54.6\% de los alumnos universitarios de Ciencias de la Actividad Física y del Deporte de España y México; enfoque superficial en el $41.3 \%$ y enfoque estratégico en solo el $4.1 \%$. En función del sexo, se apreció que son las mujeres quien tiene mayor predominio por el enfoque profundo (el $66,1 \%$ de las mujeres frente al $48.1 \%$ de los hombres) ${ }^{1}$.

En un estudio titulado llevado a cabo en 11 grupos de diferentes licenciaturas de la Universidad Politécnica de Valencia, donde realizaron seguimiento durante dos años y cuatro mediciones, reportaron que los alumnos presentan un valor mucho más alto en el enfoque profundo que en el enfoque superficial en los cuatro momentos de medida ${ }^{7}$.

En Chile, en un estudio colaborativo con España, se reportó que el $82 \%$ de los estudiantes de la licenciatura en Educación Diferencial adoptaron el enfoque profundo; el 81\% de los estudiantes de licenciatura en Educación Física, Deportes y Recreación se sitúan en el enfoque profundo, mientras que en la licenciatura en Pedagogía en Educación Parvularia y Básica para el primer ciclo predomina el enfoque profundo sobre el superficial, teniendo para el primero un $88.88 \%$ de los casos y para el segundo un $11.12 \%$ del total de la muestra ${ }^{8}$.
Finalmente, en el estudio realizado en la Universidad Mayor Temuco, en Chile, el enfoque predominante de los estudiantes de primer año de cada una de las carreras (Terapia Ocupacional, Educación Diferencial y Psicología) fue el profundo con intensidad media?.

Cabe señalar que en los estudios anteriores se empleó el cuestionario R-SPQ-2F (Revised Two Factor Study Process Questionnaire), modificado en 2001 a 20 ítems, el cual permite evaluar los enfoques de aprendizaje; distinguiendo los motivos y las estrategias que subyacen en cada uno de los enfoques de aprendizaje (superficial, profundo y mixto $)^{10,11}$. Es un instrumento utilizado en estudiantes de nivel universitario y en profesionales de la salud ${ }^{12}$.

La especialidad en Medicina familiar tiene la singularidad de no profundizar en un campo específico, sino se manejan en la transdisciplinariedad de las diferentes especialidades médicas; a diferencia de otras especialidades que ganan en la profundidad de un conocimiento o área específica, pero pierden en la transversalidad. Ante todo, el médico familiar, al ser más transdisciplinario, debe desarrollar las competencias necesarias para atender todas y cada una de las áreas de la medicina, desde lo individual hasta el ámbito familiar. Su objeto de estudio son las interacciones del proceso salud-enfermedad del individuo ${ }^{13,14}$.

El presente estudio permite conocer la forma y el grado de profundidad acerca de cómo aprenden los alumnos que cursan la especialidad en Medicina familiar, y mediante sus resultados proponer estrategias que permitan mejorar la calidad de la formación de los futuros especialistas y por consiguiente la calidad de la atención a los derechohabientes.

\section{MATERIAL Y MÉTODOS}

Se realizó un estudio transversal analítico en 131 médicos residentes de la especialidad de Medicina familiar adscritos a las tres sedes de la Delegación Guanajuato durante el ciclo escolar 2017-2018. Se consideró el 
total de los médicos residentes, por lo que no se realizó cálculo de tamaño de muestra. Se excluyeron los residentes que no aceptaron participar libremente y aquellos que se encontraban de vacaciones, licencia, servicio social o que estuvieran incapacitados laboralmente; también se eliminaron los que no contestaron completamente la encuesta.

Se acudió a las tres sedes de la residencia de Medicina familiar, se les hizo la invitación a participar en el estudio, explicándoles el objetivo y los alcances del mismo, obteniendo la firma del consentimiento informado. Para la determinación de los enfoques de aprendizaje se empleó el Cuestionario R-SPQ-2F en su versión en castellano, que consta de 20 ítems, muestra una estructura de dos escalas, de 10 ítems para el enfoque profundo y el enfoque superficial, respectivamente. Diseñado por un grupo de investigadores en 2001, se le realizaron pruebas de validez de contenido por ronda de expertos y de constructo por análisis factorial en un grupo conformado por estudiantes de psicología y otro de población en general; tiene una fiabilidad alfa de Cronbach de 0.73 para enfoque profundo y de 0.64 para enfoque superficial.

Los datos recabados se registraron en una hoja del programa Office Excel $^{\oplus}$ y el tratamiento estadístico se realizó mediante el ${\text { programa } \text { SPSS }^{\circledast} \text { v21 para Windows }}^{\oplus}$, considerando el uso de medidas de tendencia central para variables cuantitativas, tablas de frecuencia y porcentajes para cualitativas, además de la prueba Chi cuadrada para evaluar la asociación entre variables.

El protocolo fue revisado y aprobado por el Comité Nacional de Investigación Científica, de la Delegación Estatal del IMSS en Guanajuato, México, con registro N. ${ }^{\circ}$ $\mathrm{R}-2017-785022$. La presente investigación no comportó riesgo para los participantes y se realizó de acuerdo con la declaración de Helsinki actualizada en la 64. ${ }^{\text {a }}$ Asamblea General del 2013, el Reglamento de la Ley General de Salud publicada en el DOF 02-04-2014 y el Título quinto «Investigación para la salud», Capítulo único de la Ley
General de Salud publicada en el DOF 27-01-2017, en materia de investigación para la salud, vigentes en México.

\section{RESULTADOS}

Se incluyeron 131 residentes de la especialidad en Medicina familiar adscritos a la Delegación Guanajuato del IMSS (mujeres: 58.5\%, hombres: $41.5 \%$ ). El promedio de edad fue de $29.78 \pm 3.13$ años. El $80 \%$ de la muestra con formación previa en escuelas públicas; el $45.4 \%$ cursaban el primer grado, el $37.4 \%$ el segundo y el $17.2 \%$ el tercero. En el 91.6\% se identificó enfoque profundo, superficial en el 5.3\% y mixto en el 3.1\%.

Fueron excluidos 13 residentes debido a la deficiente cumplimentación de las encuestas.

Al analizar el tipo de enfoque de acuerdo con la sede se encontró un mayor predominio del enfoque profundo en las sedes en las que cuentan con todos los Servicios y se evita la rotación por sedes alternas, con una diferencia estadísticamente significativa (enfoque profundo, $\mathrm{p}=0.005$ y enfoque superficial, $\mathrm{p}=0.032)($ Tabla 1$)$.

De los médicos que presentaron un enfoque de aprendizaje superficial, 4 (3.05\%) egresaron de escuela pública y $2(1.52 \%)$ de escuela privada (Tabla 2).

\section{DISCUSIÓN}

En este estudio se presenta un análisis sobre los enfoques de aprendizaje que los médicos residentes de la especialidad de Medicina familiar de tres sedes de la Delegación Guanajuato emplean durante su proceso formativo, desde el momento en que asumen el compromiso para llevar a cabo y realizar diversas tareas académicas y asistenciales que les son encomendadas.

La mayoría de los residentes encuestados mostró un predominio del enfoque de aprendizaje profundo; se encontró una diferencia estadísticamente significativa a favor de la sede donde la rotación por los diferentes Servicios se lleva a cabo en la sede y una sola subsede, a diferencia de las dos sedes del curso que cuenta con más de 
Tabla 1. Enfoques de aprendizaje por sede hospitalaria

\begin{tabular}{|c|c|c|c|c|c|c|c|c|c|}
\hline \multirow[t]{3}{*}{ Sede } & \multirow[t]{3}{*}{ Grado escolar } & \multicolumn{6}{|c|}{ Enfoques de aprendizaje } & \multirow{2}{*}{\multicolumn{2}{|c|}{ Total }} \\
\hline & & \multicolumn{2}{|c|}{ Superficial } & \multicolumn{2}{|c|}{ Mixto } & \multicolumn{2}{|c|}{ Profundo } & & \\
\hline & & $\mathbf{n}$ & $\%$ & $\mathbf{n}$ & $\%$ & $\mathbf{n}$ & $\%$ & $\mathbf{n}$ & $\%$ \\
\hline \multirow[t]{3}{*}{1} & Primer año & 1 & 4.4 & 0 & 0 & 22 & 95.6 & 23 & 100 \\
\hline & Segundo año & 2 & 11.1 & 0 & 0 & 16 & 88.9 & 18 & 100 \\
\hline & Tercer año & 1 & 10 & 1 & 10 & 8 & 80 & 10 & 100 \\
\hline \multirow[t]{3}{*}{2} & Primer año & 0 & 0 & 0 & 0 & 14 & 100 & 14 & 100 \\
\hline & Segundo año & 0 & 0 & 0 & 0 & 14 & 100 & 14 & 100 \\
\hline & Tercer año & 0 & 0 & 0 & 0 & 6 & 100 & 6 & 100 \\
\hline \multirow[t]{3}{*}{3} & Primer año & 1 & 4.5 & 1 & 4.5 & 20 & 91 & 22 & 100 \\
\hline & Segundo año & 0 & 0 & 1 & 5.8 & 16 & 94.2 & 17 & 100 \\
\hline & Tercer año & 1 & 17 & 0 & 0 & 5 & 83 & 6 & 100 \\
\hline \multicolumn{2}{|l|}{ Total } & 6 & 4.5 & 3 & 2.3 & 121 & 93.2 & 131 & 100 \\
\hline
\end{tabular}

Fuente: Información recabada durante la fase de campo con el uso del (uestionario R-SPQ-2F (Revised Two Factor Study Process Questionnaire).

Tabla 2. Enfoques de aprendizaje por tipo de escuela

\begin{tabular}{|l|c|c|c|c|c|c|c|c|}
\hline \multirow{2}{*}{ Escuela } & \multicolumn{9}{|c|}{ Enfoques de aprendizaje } \\
\cline { 2 - 10 } & \multicolumn{2}{|c|}{ Superficial } & \multicolumn{2}{|c|}{ Mixto } & \multicolumn{2}{c|}{ Profundo } & \multicolumn{2}{c|}{ Total } \\
\cline { 2 - 10 } & $\mathbf{n}$ & $\%$ & $\mathbf{n}$ & $\%$ & $\mathbf{n}$ & $\%$ & $\mathbf{n}$ & $\%$ \\
\hline Pública & 4 & 3.8 & 1 & 1 & 100 & 95.2 & 105 & 100 \\
\hline Privada & 2 & 7.7 & 2 & 7.7 & 22 & 84.6 & 26 & 100 \\
\hline Total & 6 & 4.6 & 3 & 2.3 & 122 & 93.1 & 131 & 100 \\
\hline
\end{tabular}

Fuente: Información recabada durante la fase de campo con el uso del (uestionario R-SPQ-2F (Revised Two Factor Study Process Questionnaire).

dos subsedes. Esto último pudiera explicarse debido a que en la primera, que cuenta con todos los Servicios, se tiene una supervisión directa del profesor titular y coordinador de educación, además de que se puede establecer una mayor comunicación con los profesores de asignatura y de clínica, situación que muchas veces se limita en las que cuentan con varias subsedes. Asimismo, el sentido de pertenencia puede influir en un mejor aprendizaje; siendo estas últimas inferencias susceptibles de ser investigadas en futuros trabajos.

Los resultados encontrados son similares a los reportados por Cumplido-Hernández, et al..$^{15}$ en el año 2004 en su estudio realizado en el Centro Médico Nacional de Occidente, quienes estudiaron 179 residentes de los 19 cursos de especialización médica y encontraron que el $60.3 \%$ tenían un enfoque profundo, en tanto que en el $39.7 \%$ el enfoque era superficial, predominando en mujeres este enfoque con un $68.4 \%$.

Por otra parte, García-Berbén ${ }^{16}$, en un estudio realizado en estudiantes de Magisterio y Psicopedagogía utilizando el cuestionario R-SPQ-2F, encontraron que el sexo y la edad influyen significativamente en el enfoque, puesto que el sexo femenino adopta más el enfoque profundo, en comparación con el sexo masculino; también observaron que a mayor edad, mayor predilección por este enfoque. 
En el estudio realizado en Colombia por Montealegre, et al. ${ }^{17}$, en estudiantes de los programas de Medicina, Enfermería y Educación física $(\mathrm{n}=112)$ durante el semestre A 2013 en la Universidad del Tolima se describe la muestra en general y por programa, en función de sus enfoques de aprendizaje. Se reporta que el $36.6 \%$ adoptaría un enfoque profundo en su ingreso a las carreras del área de la salud, mientras que el $30.4 \%$ elige un enfoque superficial y el restante $33 \%$ sigue un enfoque estratégico. Con relación a los resultados de enfoques de aprendizaje por tipo de carrera, en los estudiantes de Medicina y Educación física predomina el enfoque estratégico, mientras que en enfermería lo hace el enfoque profundo. En lo que respecta a las tres variables socioculturales (sexo, tipo de colegio y cabeza de hogar) no existe relación entre los enfoques de aprendizaje.

En la investigación llevada a cabo en la Universidad Noreste de México «Enfoques hacia el aprendizaje, motivos y estrategias de estudiantes de las carreras de enfermería, ingeniería y organización deportiva», en el cual participaron 173 estudiantes (98 hombres y 75 mujeres), se reportó una diferencia estadísticamente significativa $(\mathrm{p}<0.001)$ para las carreras de Enfermería e Ingeniería del enfoque profundo sobre el enfoque superficial, y en lo que se refiere a la carrera de Organización Deportiva no hubo diferencia estadística ( $\mathrm{p}<0.836$ ), pese al predominio del enfoque profundo sobre el enfoque superficial. En el análisis comparativo entre las tres carreras, con relación al enfoque profundo, Ingeniería obtuvo la mayor puntuación, seguida de Enfermería, sin que la diferencia fuese estadísticamente significativa ( $p<0.228)$, a diferencia de la carrera de Organización Deportiva con respecto a las dos anteriores, donde la diferencia sí fue significativa ( $p<0.003$ ), pese a tener la puntuación menor. Finalmente, la mayor puntuación para enfoque superficial se observó en la carrera de Organización Deportiva, con respecto a Ingeniería y Enfermería, con diferencia estadísticamente significativa $(p<0.0019)$ en ambos casos ${ }^{18}$.

Pérez, et al. ${ }^{19}$ realizaron un estudio en estudiantes de tercer semestre de la carrera de Medicina e identificaron que la mayoría de los estudiantes $(86.6 \%)$ presentaban un enfoque profundo de aprendizaje, en tanto que solo el $14 \%$ de ellos presentaron enfoque superficial $^{19}$.

Por la naturaleza del estudio, la mayor limitación concierne a la necesidad de conocer el comportamiento de los residentes conforme avanzan de grado escolar, con la finalidad de identificar cómo es la postura con relación a los enfoques de aprendizaje, lo que permitiría valorar desde un inicio hasta el final como se mantienen, y conforme avanzan en qué momento cambian. También es importante señalar el déficit en cuanto al análisis de la sede que no cuenta con rotación externa en otras subsedes, con respecto a las que sí tienen rotación por distintas subsedes. Los participantes correspondientes del tercer grado de residencia constituyen otro punto que resaltar, debido a que solo participó la mitad con respecto al total de alumnos de primero y segundo año, debido a los seis meses de servicio social que deben cumplir como parte del programa académico. Otra limitación a considerar es la relación del nivel académico de acuerdo con el enfoque de aprendizaje que adoptan durante el curso de la residencia de Medicina familiar. Así también, el hecho de que el proceso de aprendizaje no depende solo del enfoque, sino de otros factores contextuales como el tipo de profesores, los programas académicos, el apoyo de las autoridades, etc., los cuales pueden abrir otros estudios de investigación.

El estudio no permite evaluar la implicación que tiene el género en el tipo de enfoque ni qué habilidades son las que más se desarrollan. Así como tampoco los cambios que habría que hacer en el programa de Medicina familiar para optimizar el enfoque profundo, dado que no fue su objetivo. Sin embargo, sí da una pauta a indagar al 
respecto, por lo que se abre una línea de investigación en relación a este tema.

Dentro de las fortalezas del trabajo está la concordancia con respecto a otros estudios realizados en los que el enfoque profundo es el mayormente adoptado por los estudiantes, así como también la no existencia de diferencias en relación con aspectos socioculturales como la escuela de procedencia, además del estado civil. Se sientan las bases para el desarrollo de futuros estudios en esta línea de investigación educativa, debido a los pocos trabajos que hoy en día se han realizado en el área de la salud, específicamente a nivel de posgrados.

Partiendo de los resultados de nuestro estudio, se concluye que es imprescindible el diseño de estrategias para la preservación del enfoque profundo en los residentes, así como también promoverlo en aquellos que utilizan el enfoque superficial, con la finalidad de lograr en cada uno de ellos un aprendizaje significativo, que les permita afrontar las diversas situaciones de su entorno laboral y familiar, otorgando una atención de calidad y calidez a los derechohabientes, favoreciendo así la resolución de problemas y toma de decisiones.

\section{CONCLUSIONES}

El enfoque prevalente fue el profundo. En la sede sin rotación externa se encontró únicamente el enfoque profundo.

\section{BIBLIOGRAFÍA}

1. Ruiz Lara E, Hernández Pina F, Ureña Villanueva F. Enfoques de aprendizaje y rendimiento institucional y afectivo de los alumnos de la titulación de Ciencias de la Actividad Física y del deporte. RIE. Rev Invest Educ.. 2008;26(2):307-32.

2. Equipo docente en ABP. Facultad de Psicología. Enfoques de aprendizaje en estudiantes universitarios. Universidad de Murcia. Recuperado de: http://ocw.um.es/cc-sociales/la-metodología-de -aprendizaje-basado-en-problemas/material-de-clase-1/tema-14. pdf (último acceso 15 de agosto de 2016).
3. Santos Rego MA, Salas Silva R, Parra Arenas S. Enfoques de aprendizaje y dominancias cerebrales en estudiantes universitarios. ICE Universidad de Oviedo, Aula Abierta. 2004;(84):3-22.

4. Hernández Pina F, Hervás Avilés RM. Enfoques y estilos de aprendizaje en educación superior. Rev Orientac Psicopedag. 2005;16(2):283-99.

5. García-Perdomo HA, Elías de la Hoz G. Enfoques de aprendizaje en los estudiantes de Medicina de una universidad en la ciudad de Cali (Colombia). Educación y Humanismo. 2015;17(28):30-46.

6. López-Alonso Al, López-Aguado M, González-Millán I, Fernández Martínez ME. El ocio y los enfoques de aprendizaje en estudiantes universitarios de enfermería. RIE. Rev Invest Educ. 2012;30(1):53-70.

7. Gallardo López B, Suárez Rodríguez J, Jiménez Rodríguez $M$, Rodríguez Monzonís M. Enfoques de aprendizaje en estudiantes universitarios. Su evolución durante los dos primeros cursos. Ponencia presentada en el VII Congreso Internacional de Docencia Universitaria e Innovación. 4-6 de julio de 2012. Universidad Pompeu Fabra, Barcelona, España [Internet]. Barcelona:Universidad Pompeu Fabra [último acceso: 28 de abril de 2016]. Disponible en: https://www.cidui.org/congressos-cidui/vii-congres-cidui-2012

8. Hernández Piña F, Arán Jara A, Salmerón Pérez H. Enfoques de aprendizaje y metodologías de enseñanza en la universidad. Rev Iberoam Educ. 2012;60(3).

9. Arán Jara M, Ortega Triviños M. Enfoques de aprendizaje y hábitos de estudio en estudiantes universitarios de primer año de tres carreras de la Universidad Mayor Temuco, Chile 2011. Revista Educativa Hekademos. 2012;11(5):37-46.

10. González Geraldo JL, del Rincón Igea B, del Rincón Igea DA. Estructura latente y consistencia interna del R-SPQ-2F: reinterpretando los enfoques de aprendizaje en el EEES. RIE Rev Invest Educ. 2011;29(2):277-93.

11. Merino Soto $C$, Kumar Pradham R. Validación estructural del R-SPQ-2F: Un análisis factorial confirmatorio. Revista Digital de Investigación en Docencia Universitaria [Internet]. 2013 [último acceso: 30 de junio de 2016];7(1):112-27. Disponible en: DOI: http://dx.doi.org/10.19083/ridu.7.190

12. Biggs J, Kember D, Leung D. The revised two-factor study process questionnaire: R-SPQ-2F. Br J Educ Psychol. 2001;71:133-49.

13. García Morales VA, Gómez Soto V, Vargas Machado G. Formación del médico familiar, En: Dávila Torres J, Garza Sagástegui MG, editores .Medicina Familiar. Academia Mexicana de Cirugía A.C. México :Alfil.;2013., p13-22.

14. Farfán Salazar G. Perfil profesional del médico familiar en México. Medicina familiar en la práctica. Documentos y presentaciones. México. Alfil; 2015. p 5-15.

15. Cumplido-Hernández G, Campos-Arciniega MF, Chávez-López A, García-Pérez V. Enfoques de aprendizaje que utilizan médicos residentes en el desarrollo de una especialidad médica. Rev Med Inst Mex Seguro Soc. 2006;44(4):321-8.

16. García Berbén AB. Estudio de los enfoques de aprendizaje en estudiantes de Magisterio y Psicopedagogía. Electronic Journal of Research in Educational Psychology [Internet]. 2005;3(2):10926 [último acceso: 12 de marzo de 2018]. Disponible en: http:// www.redalyc.org/articulo.oa?id=293121927006

17. Montealegre G, Núñez ML, Salgado D. Enfoques de aprendizajes y variables de orden sociocultural en estudiantes de una institución de educación superior en Colombia. Act Med Col. 2014;39(4):368-77.

18. Esquivel Cruz J, Rodríguez Nieto MC, Padilla Montemayo, VM. Enfoques hacia el aprendizaje, motivos y estrategias de estudiantes de las carreras de enfermería, ingeniería y organización deportiva. Rev Pedagog - Esc Educ, Univ Cent Venez. 2009;30(87):309-31.

19. Pérez Riveros E, Bernal M, González N. Prevalencia de los enfoques de aprendizaje en estudiantes de fisiología médica: cuestionario de proceso de estudio revisado de dos factores (R-SPQ-2F). Biosalud. 2011;10(2):37-47. 